\title{
Optimal three dimensional aircraft terrain following and collision avoidance
}

\section{T. Sharma* $\quad$ P. Williams ${ }^{\dagger} \quad$ C. Bil ${ }^{\ddagger} \quad$ A. Eberhard ${ }^{\S}$}

(Received 16 March 2006, revised 15 April 2007)

\begin{abstract}
Military aircraft must often fly in close proximity to terrain. In this article, optimal terrain following is considered as a minimax optimal control problem, which is solved using direct transcription of the continuous optimal control problem. Within a very general framework for solving such problems, we transform the nonsmooth cost function into a constrained nonlinear programming problem. In the formulation, we solve for optimal collision avoidance manoeuvres. To ensure smooth derivatives of general three dimensional terrain, it is approximated using B-splines. A receding horizon tracking controller tracks the optimal trajectories with disturbances to the aircraft model and initial conditions.
\end{abstract}

*School of Aerospace, Mechanical, and Manufacturing Engineering, RMIT University, Bundoora, AustraliA. mailto:S3075886@student.rmit.edu.au

${ }^{\dagger}$ School of Aerospace, Mechanical, and Manufacturing Engineering, RMIT University, Bundoora, Australia. mailto:paul.williams@rmit.edu.au

${ }^{\ddagger}$ Sir Lawrence Wackett Centre, RMIT University, Melbourne, Australia.

$\S$ Department of Mathematics, RMIT University, Melbourne, Australia.

See http://anziamj.austms.org.au/V47EMAC2005/Sharma for this article, (C) Austral. Mathematical Soc. 2007. Published June 26, 2007. ISSN 1446-8735 


\section{Contents}

1 Introduction

C696

2 Dynamical model

C697

3 Terrain following and collision avoidance problem

C699

4 Solution via direct transcription

C701

4.1 Terrain modelling . . . . . . . . . . . . . . .

C702

5 Feedback tracking control

C702

6 Numerical results

C704

7 Conclusions

C710

References

C710

\section{Introduction}

Military aircraft are often required to fly close to terrain to help avoid radar detection. This is not always easy for a pilot to achieve because the manoeuvres are often complex and the aircraft must function close to the operational constraints [1]. Various approaches have been used for planning trajectories in different terrain environments. Some of these are based purely on kinematic considerations [2], while others incorporate the aircraft dynamics, but consider only two dimensional motion [3]. In most cases where optimal control of the aircraft is required, a weighted minimum-time and minimumaltitude criterion has been used. In this work, an approach for planning aircraft trajectories for maneuvering close to arbitrary three dimensional terrain is developed. The problem is formulated as a nonlinear minimum-time/ 
minimax altitude optimal control problem. The optimal control problem is formulated as a nonlinear programming problem, and the nonsmooth cost function is transformed into a smooth cost by introducing additional inequality constraints. A feedback tracking controller tracks the optimal reference trajectories.

\section{Dynamical model}

For the purposes of flight path control design, it is sufficient to treat only the translational motion of the aircraft. In this context, the aircraft angle of attack $\alpha$ and bank angle $\phi$ are treated as pseudocontrol inputs, together with the throttle $\eta$. The aircraft equations of motion are expressed in a velocity coordinate frame attached to the aircraft. The kinematic equations of the aircraft are

$$
\begin{aligned}
& \dot{x}=V \cos \gamma \cos \chi+W_{x}, \\
& \dot{y}=V \cos \gamma \sin \chi+W_{y}, \\
& \dot{z}=V \sin \gamma+W_{z},
\end{aligned}
$$

where $V$ is the aircraft velocity relative to the wind, $\gamma$ is the flight-path angle, $\chi$ is the heading angle, $W_{x}, W_{y}$ and $W_{z}$ are components of the wind velocity in the inertial frame, and $(x, y, z)$ are the aircraft coordinates.

The dynamical equations are

$$
\begin{aligned}
\dot{V}= & \frac{T \cos (\alpha+\varepsilon)-D}{m}-g \sin \gamma-\dot{W}_{x} \cos \gamma \cos \chi \\
& -\dot{W}_{y} \cos \gamma \sin \chi-\dot{W}_{z} \sin \gamma, \\
\dot{\chi}= & \frac{[L+T \sin (\alpha+\varepsilon)] \sin \phi}{m V \cos \gamma}+\frac{\dot{W}_{x} \sin \chi}{V \cos \gamma}-\frac{\dot{W}_{y} \cos \chi}{V \cos \gamma}, \\
\dot{\gamma}= & \frac{[L+T \sin (\alpha+\varepsilon)] \cos \phi}{m V}-\frac{g}{V} \cos \gamma+\frac{\dot{W}_{x} \sin \gamma \cos \chi}{V} \\
& +\frac{\dot{W}_{y} \sin \gamma \sin \chi}{V}-\frac{\dot{W}_{z} \cos \gamma}{V},
\end{aligned}
$$


where $T=\eta T_{\max }$ is the thrust, $T_{\max }$ is the maximum available thrust, $m$ is the aircraft mass, $D=q S C_{D}$ is the drag, $g$ is the acceleration due to gravity, $L=q S C_{L}$ is the lift, $\varepsilon$ is the thrust angle relative to the zero lift axis, $q=\frac{1}{2} \rho V^{2}$ is the dynamic pressure, $\rho$ is the air density, and $S$ is the aircraft reference wing area.

For convenience, the equations of motion are nondimensionalised using the following strategy: $\bar{V}=V / V_{s}, \tau=g t / V_{s}, \bar{x}=g x / V_{s}^{2}, \bar{y}=g y / V_{s}^{2}$, $\bar{z}=g z / V_{s}^{2}, d() / d t=g / V_{s}[d() / d \tau]$, and $d^{2}() / d t^{2}=\left(g / V_{s}\right)^{2}\left[d^{2}() / d \tau^{2}\right]$, where $V_{s}$ is the speed of sound at sea level. This scaling essentially reduces the aircraft velocity to the Mach number. For the sake of brevity, the final form of the equations are omitted. The equations of motion presented above are rather general, and to completely specify the motion for a particular aircraft some aerodynamic data is necessary. We used data corresponding to the F4 supersonic military jet. The data was originally presented by Bryson et al. $[4,5]$.

The maximum thrust $T_{\max }$ is expressed in units of $1000 \mathrm{lb}$, and is a function of Mach number $\bar{V}$ and altitude $h$ in units of $10000 \mathrm{ft}$,

$$
T_{\max }=\left[\begin{array}{c}
1 \\
\bar{V} \\
\bar{V}^{2} \\
\bar{V}^{3} \\
\bar{V}^{4}
\end{array}\right]^{\top}\left[\begin{array}{rrrrr}
30.21 & -0.668 & -6.877 & 1.951 & -0.1512 \\
-33.80 & 3.347 & 18.13 & -5.865 & 0.4757 \\
100.80 & -77.56 & 5.441 & 2.864 & -0.3355 \\
-78.99 & 101.40 & -30.28 & 3.236 & -0.1089 \\
18.74 & -31.60 & 12.04 & -1.785 & 0.09417
\end{array}\right]\left[\begin{array}{c}
1 \\
h \\
h^{2} \\
h^{3} \\
h^{4}
\end{array}\right]
$$

The lift and drag coefficients for $\bar{V}<1.15$ are

$$
\begin{aligned}
& C_{L}=C_{L_{\alpha}} \alpha, \\
& C_{L_{\alpha}}=3.44+1 / \cosh ^{2}\left(\frac{\bar{V}-1}{0.06}\right), \\
& C_{D}=0.013+0.0144\left[1+\tanh \left(\frac{\bar{V}-0.98}{0.06}\right)\right]
\end{aligned}
$$




$$
+\left(0.54+0.15\left[1+\tanh \left(\frac{\bar{V}-0.9}{0.06}\right)\right]\right) C_{L_{\alpha}} \alpha^{2}
$$

\section{Terrain following and collision avoidance problem}

We assume that the terrain being followed is available from a known terrain database. Terrain following necessarily entails collision avoidance because the optimal aircraft flight path must be free of obstacles while maintaining the aircraft as close to the terrain as possible. In practice a set clearance height $h_{c}$ is used so that the aircraft maintains a safe tolerance above the terrain to compensate for flight control errors or other disturbances. A variety of different techniques have been used to plan such trajectories. Typically, a weighted minimum time/minimum $l_{2}$-norm of the aircraft distance from the terrain is used as a performance index. However, the performance of the aircraft then necessarily depends on the relative weighting of the two costs. In addition, this choice of cost does not keep the aircraft as close to the terrain as possible, but rather minimizes the distance only in an "average" sense. Consider the aircraft height above the terrain,

$$
h(t) \triangleq z(t)-h_{c}-h_{T}[x(t), y(t)]
$$

where $h_{T}[x(t), y(t)]$ is the height of the terrain at the aircraft position. For a scalar valued function, $f: \mathbb{R} \supseteq \Omega \rightarrow \mathbb{R}$, define the $l_{p}$-norm of $f$ as

$$
\|f\|_{l_{p}} \triangleq\left(\int_{\Omega}|f(t)|^{p} d t\right)^{1 / p}
$$

A potential cost function for minimizing distance from the terrain is

$$
J_{1}=\int_{t_{0}}^{t_{f}}|h(t)| d t=\|h(t)\|_{l_{1}}
$$


whereas the typical cost function employed for terrain following is

$$
J_{2}=\int_{t_{0}}^{t_{f}}[h(t)]^{2} d t=\|h(t)\|_{l_{2}}^{2} .
$$

The costs $J_{1}$ and $J_{2}$ can produce drastically different solutions for different problems. However, $J_{1}$ is not often used for terrain following because it is non-differentiable with respect to the states. For this reason, some function involving $J_{2}$ is used. This does not generally give the best performance for terrain following. Another choice that gives much better solutions for hugging terrain is to minimise the $l_{\infty}$-norm:

$$
\|f(t)\|_{l_{\infty}} \triangleq \max |f(t)| .
$$

To combine the two measures of minimum time and terrain hugging, we employ the nonlinear cost

$$
J=t_{f} \max |h(t)| .
$$

The rationale for this choice is that the cost minimises the maximum distance of the aircraft from the terrain and hence defines the terrain envelope that the aircraft flies within for a given flight time. Because the maximum distance from the clearance height is unlikely to be identically zero, then the cost is also an appropriate minimum-time cost. Hence, the cost properly incorporates both objectives without the need to weight one over the other. In a sense, this makes the problem one of terrain following in four dimensions because the solution seeks the fastest and closest aircraft trajectory through the complex terrain to reach the desired downrange.

The control problem is to take the aircraft from a given initial state $[\bar{x}(0), \bar{y}(0), \bar{z}(0), \bar{V}(0), \chi(0), \gamma(0)]=\left[\bar{x}_{0}, \bar{y}_{0}, \bar{z}_{0}, \bar{V}_{0}, \chi_{0}, \gamma_{0}\right]$ to a final state $\left[\bar{x}\left(t_{f}\right)\right.$, $\left.\bar{y}\left(t_{f}\right), \bar{z}\left(t_{f}\right), \bar{V}\left(t_{f}\right), \chi\left(t_{f}\right), \gamma\left(t_{f}\right)\right]=\left[\bar{x}_{f}\right.$, free, free, $\left.\bar{V}_{f}, \chi_{f}, \gamma_{f}\right]$ to minimise the cost function in equation (14) subject to the dynamical constraints in equations (1) through (4), and the path constraints

$$
z(t) \geq h_{c}+h_{T}[x(t), y(t)] .
$$


However, the use of the angle of attack and bank angle as pseudocontrols can theoretically lead to instantaneous changes in the controls, which would be impossible to achieve in practice. Hence, the real aircraft could not follow the trajectory 'precisely' and may crash if such a sudden change occurs very close to the clearance height. To account for limited rigid body reorientation of the aircraft, we constrain the rate of angle of attack and bank angle to

$$
\dot{\alpha}_{\min } \leq \dot{\alpha} \leq \dot{\alpha}_{\max } \quad \text { and } \quad \dot{\phi}_{\min } \leq \dot{\phi} \leq \dot{\phi}_{\max } .
$$

For the control design, the rate of angle of attack and rate of bank are treated as the new control variables, and the constraints on the actual angle of attack and bank angle become first order state constraints

$$
\alpha_{\min } \leq \alpha \leq \alpha_{\max } \quad \text { and } \quad \phi_{\min } \leq \phi \leq \phi_{\max } .
$$

\section{Solution via direct transcription}

The solution to the optimal control problem defined in the preceding section would typically involve the application of an indirect method utilising Pontryagin's Maximum Principle, or similar variant for minimax optimal control problems. Instead of performing this laborious task of deriving the necessary conditions and trying to solve the resulting problem, direct transcription methods combine the latest advances in numerical methods with optimisation algorithms to automate the procedure. In this view, one discretises the continuous problem by an appropriate scheme to convert it into a large scale parameter optimisation problem. This process is automated in the software DIRECT [6], which is implemented in the MATLAB environment. DIRECT has a range of discretisation methods available, including HermiteSimpson, Legendre pseudospectral, Gauss-Lobatto quadrature, among others. DIRECT uses the sequential quadratic programming software SNOPT to solve the resulting parameter optimisation problem. We use a Gauss-Lobatto quadrature discretisation [7]. 
To incorporate the nonsmooth cost defined in equation (14) within the framework of DIRECT, it is necessary to introduce the additional parameter $p$ and the inequality constraints

$$
h(t) \leq p, \quad h(t) \geq-p \quad \text { and } \quad 0 \leq p \leq p_{\max } .
$$

This allows the cost function to be written as a smooth cost as

$$
J=p t_{f} .
$$

\subsection{Terrain modelling}

The terrain used in the optimisation problem is modelled using a matrix of elevation data provided by a terrain generation program. We constructed our own terrain models that are representative of complex terrain. The terrain model is provided as a set of $x$ and $y$ coordinates, and a matrix of $z$ coordinates representing the elevation. In the solution technique, interpolated values of the elevation data are needed. In addition, gradients of the constraints are calculated by way of finite differences. This means that smooth derivatives of the terrain data are required for the solution algorithm to be effective. It is possible to provide $C^{2}$ continuity by approximating the data with a tensor product cubic B-spline of the form

$$
h_{T}(x, y)=\sum_{i=1}^{n_{1}} \sum_{j=1}^{n_{2}} c_{i, j} B_{i}(x) B_{j}(y),
$$

where $c_{i, j}$ are a set of coefficients and $B_{i}(x)$ and $B_{j}(y)$ form the basis for cubic B-splines [8].

\section{$5 \quad$ Feedback tracking control}

To ensure that the aircraft accurately follows the optimal trajectory, it is necessary to provide feedback control. This is used to compensate for errors 
in the aircraft parameters as well as disturbances due to gusts or errors in the initial aircraft speed, flight path, and so on. Although ultimately the controller must be flown using a real aircraft, we utilise the same trajectory equations to simulate possible disturbances to the system and to study the sensitivity of the optimal manoeuvres. To this end, we employed a linear receding horizon control strategy.

Denoting the perturbed aircraft state vector by

$$
\delta \mathbf{x}(t)=[\delta \bar{x}, \delta \bar{y}, \delta \bar{z}, \delta \bar{V}, \delta \gamma, \delta \chi, \delta \alpha, \delta \phi, \delta \eta]^{\top}
$$

and the perturbed control vector as $\delta \mathbf{u}(t)=[\delta \dot{\eta}(t), \delta \dot{\alpha}(t), \delta \dot{\phi}(t)]^{\top}$, then the feedback tracking control problem is: Find the perturbed state-control pair $[\delta \mathbf{x}(t), \delta \mathbf{u}(t)]$ to minimise the quadratic cost

$\delta J=\frac{1}{2} \delta \mathbf{x}^{\top}\left(t+T_{h}\right) \mathbf{S}_{f} \delta \mathbf{x}(t+T)+\frac{1}{2} \int_{t}^{t+T}\left[\delta \mathbf{x}^{\top}(t) \mathbf{Q} \delta \mathbf{x}(t)+\delta \mathbf{u}^{\top}(t) \mathbf{R} \delta \mathbf{u}(t)\right] \mathrm{d} t^{*}$,

subject to the linearised dynamical equations

$$
\delta \dot{\mathbf{x}}=\mathbf{A}(t) \delta \mathbf{x}+\mathbf{B}(t) \delta \mathbf{u},
$$

and the initial conditions

$$
\delta \mathbf{x}\left(t^{*}=t\right)=\delta \mathbf{x}(t)
$$

where $\mathbf{A} \in \mathbb{R}^{9 \times 9}$ is the system state influence matrix, $\mathbf{B} \in \mathbb{R}^{9 \times 3}$ is the control influence matrix, $\mathbf{Q} \in \mathbb{R}^{9 \times 9}$ is a positive semi-definite weighting matrix that penalises the deviations of the perturbed states, $\mathbf{R} \in \mathbb{R}^{3 \times 3}$ is a positive definite weight matrix that penalises the deviations of the perturbed controls, and $\mathbf{S}_{f} \in \mathbb{R}^{9 \times 9}$ is a positive semi-definite terminal weight matrix that penalises the deviations of the perturbed states at the end of the future horizon $T_{h}$. Note the distinction between the actual system states and controls defined as a function of $t$, and the predicted states and controls defined as a function of the time $t^{*}$. The resulting control law can be expressed as a 
linear feedback control law with a set of time varying gains. The closed loop stability of the system depends on the selection of parameters in the feedback controller, but the feedback controller is only valid within the linear range along the optimal trajectory. In the case of very large parameter uncertainties, it is necessary to introduce additional state variables representing the integral of the error of selected states. In this way, integral control action can be incorporated into the feedback controller to increase robustness. However, these issues are beyond the scope of this work.

\section{$6 \quad$ Numerical results}

An optimal aircraft trajectory was determined using 129 nodes along the trajectory, with the following parameters: $\alpha_{\min }=-10^{\circ}, \alpha_{\max }=20^{\circ}, \phi_{\min }=$ $-70^{\circ}, \phi_{\max }=70^{\circ}, \dot{\alpha}_{\min }=-8^{\circ} / \mathrm{sec}, \dot{\alpha}_{\max }=8^{\circ} / \mathrm{sec}, \dot{\phi}_{\min }=-15^{\circ} / \mathrm{sec}, \dot{\phi}_{\max }=$ $15^{\circ} / \mathrm{sec}, \eta_{\min }=0.1, \eta_{\max }=0.9, \dot{\eta}_{\min }=-0.25 / \mathrm{sec}, \dot{\eta}_{\max }=-0.25 / \mathrm{sec}$. Note that the bounds on the throttle are set to allow $10 \%$ tolerance for feedback. The initial downrange is $-9 \mathrm{~km}$, the final downrange is $9 \mathrm{~km}$, the initial and final Mach numbers are set to 0.7, and the initial and final flight path and heading angles are set to zero. The initial cross-range is set to $3 \mathrm{~km}$, and the final cross-range is free. In addition, the initial and final altitudes are free, and a cross-wind of $W_{y}=6 \mathrm{~m} / \mathrm{s}$ is included.

For feedback control, the parameters

$$
\begin{array}{ll} 
& \mathbf{Q}=\operatorname{diag}[1000,1000,10000,10,10,10,10,10,10], \\
& \mathbf{R}=\operatorname{diag}[1,1,1] \\
& \mathbf{S}_{f}=\operatorname{diag}\left[10^{5}, 10^{5}, 10^{8}, 10^{2}, 10^{2}, 10^{2}, 2,1,1\right] \\
\text { and } \quad & T_{h}=10 \mathrm{~s}
\end{array}
$$

These weighting matrices are a design choice and are selected to more heavily weight deviations from the optimal aircraft position, with higher weighting 
given to the aircraft altitude. Different weightings will naturally result in different closed loop performance. However, studies of different weighting matrices is beyond the scope of this article. Numerical simulations of the closed loop trajectories were performed with errors in the initial conditions dispersed randomly in the bounds: $\pm 2 \%$ error in position, $\pm 10 \%$ error in velocity, \pm 2 degrees error in flight path and heading angles, and $\pm 10 \%$ errors in the first aerodynamic terms in equations (7) and (8). Numerical results are shown in Figures 1 to 4 for 15 simulation runs. The flight time is $113.13 \mathrm{sec}-$ onds and the maximum altitude above the terrain is $42.28 \mathrm{~m}$, which is only $2.28 \mathrm{~m}$ above the set clearance height. Thus, the aircraft maneuvers within a $2.28 \mathrm{~m}$ envelope. Figure 1 shows the terrain map together with the optimal open loop trajectory. This illustrates the relative complexity of the terrain and that the aircraft performs both lateral and longitudinal manoeuvres in order to follow the terrain closely. Figure 2 shows the closed loop variations in altitude above the terrain. The initial disturbances cause considerable variations in the altitude (up to $95 \mathrm{~m}$ from the terrain), but the aircraft does not collide with the terrain. The errors damp out well despite the uncertainty in the aerodynamic parameters. Figure 3 shows the open loop and closed loop Mach number and applied throttle. Evidently, the closed loop trajectories require significant control effort to correct the trajectories due to the initial errors. In some simulations, the throttle and throttle rate are both saturated. The dispersions in throttle later in the trajectory are due to the parameter uncertainties. Finally, Figure 4 shows the angle of attack and bank angle. This figure illustrates that the parameter uncertainties have little effect on the bank angle, and a relatively minor effect on the angle of attack. Although not shown here, the rate of angle of attack is saturated in many of the closed loop trajectories due to initial disturbances. 


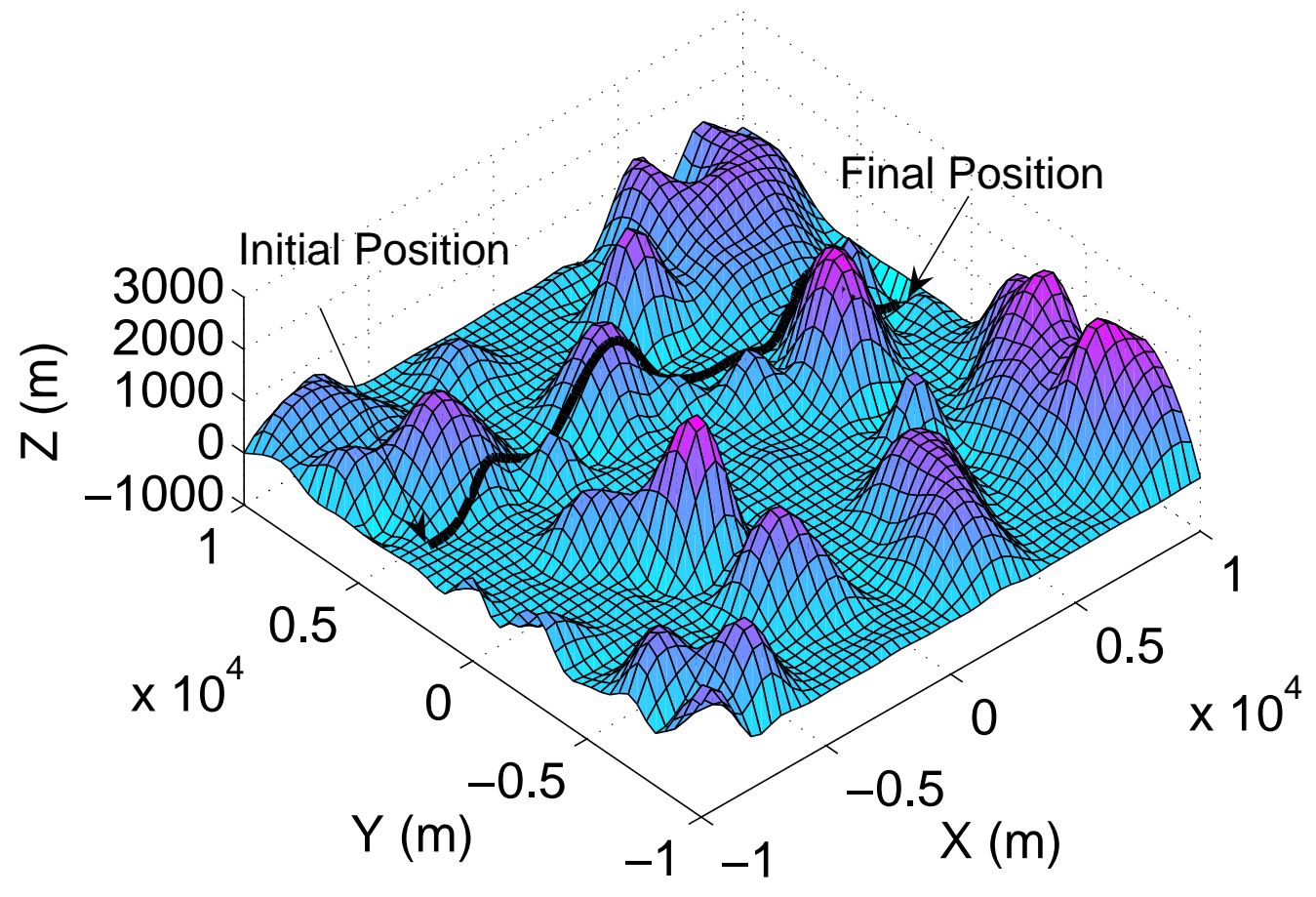

FiguRE 1: Optimal aircraft trajectory over three dimensional terrain. 


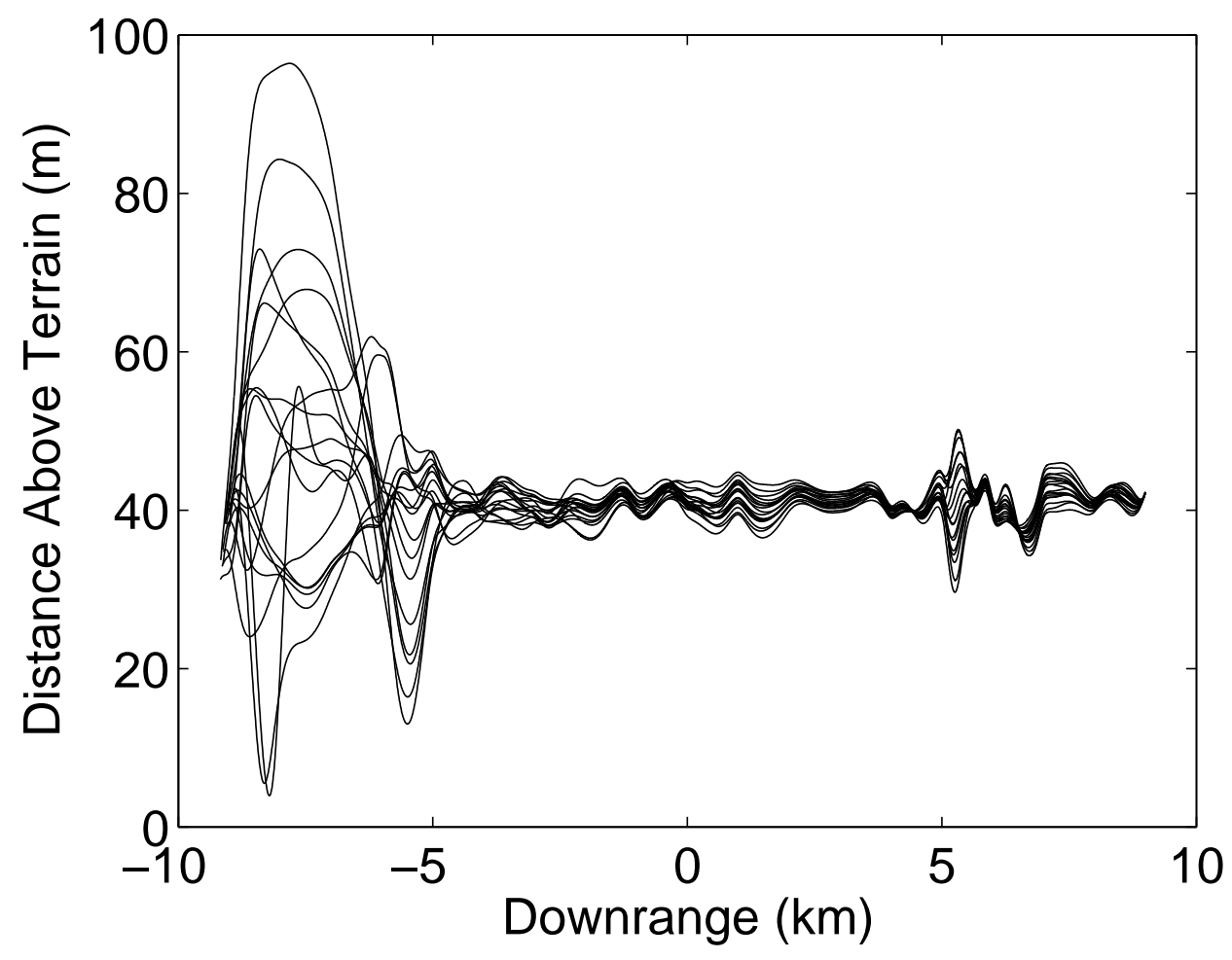

Figure 2: Closed loop variations in altitude. 

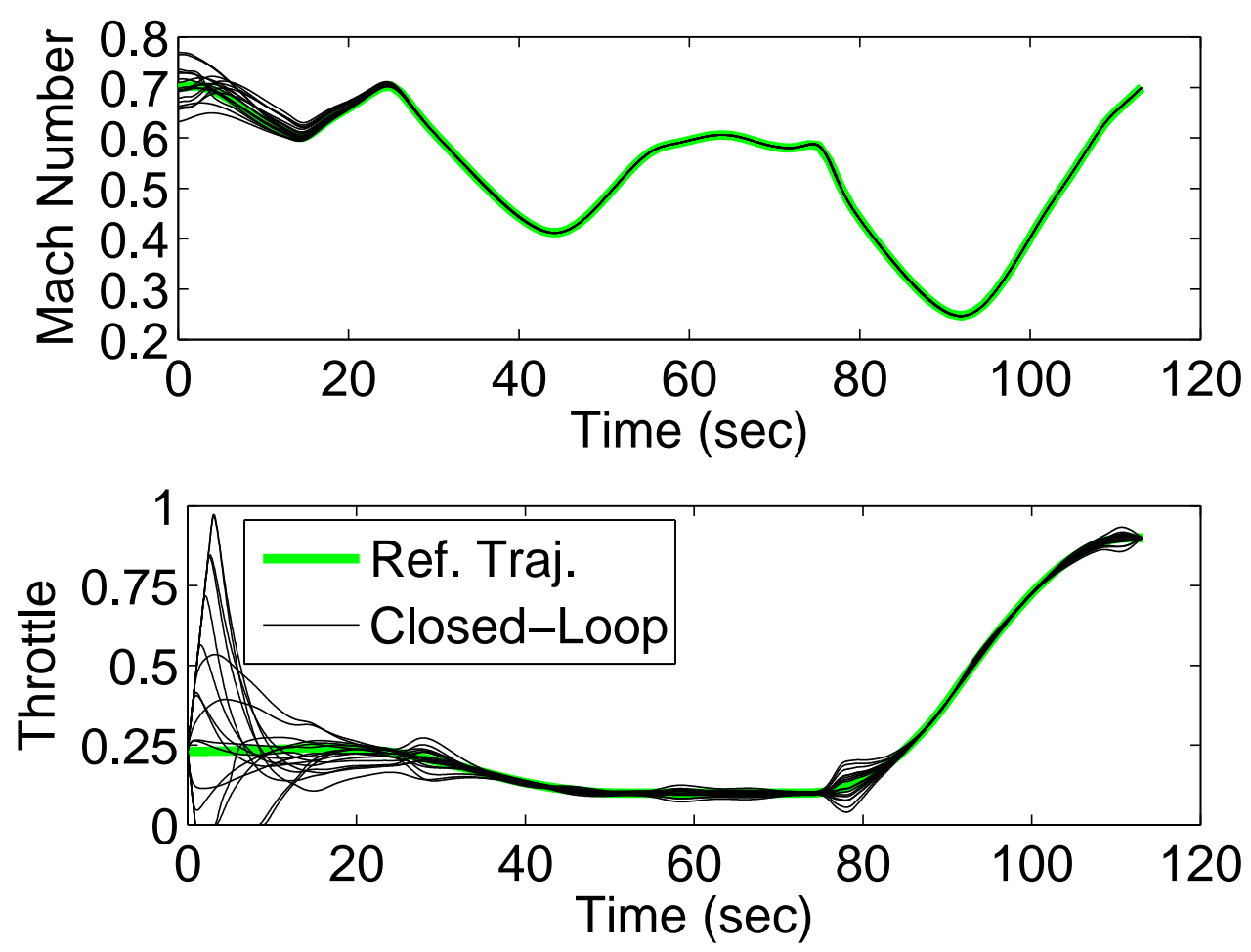

Figure 3: Closed loop Mach number and thrust. 

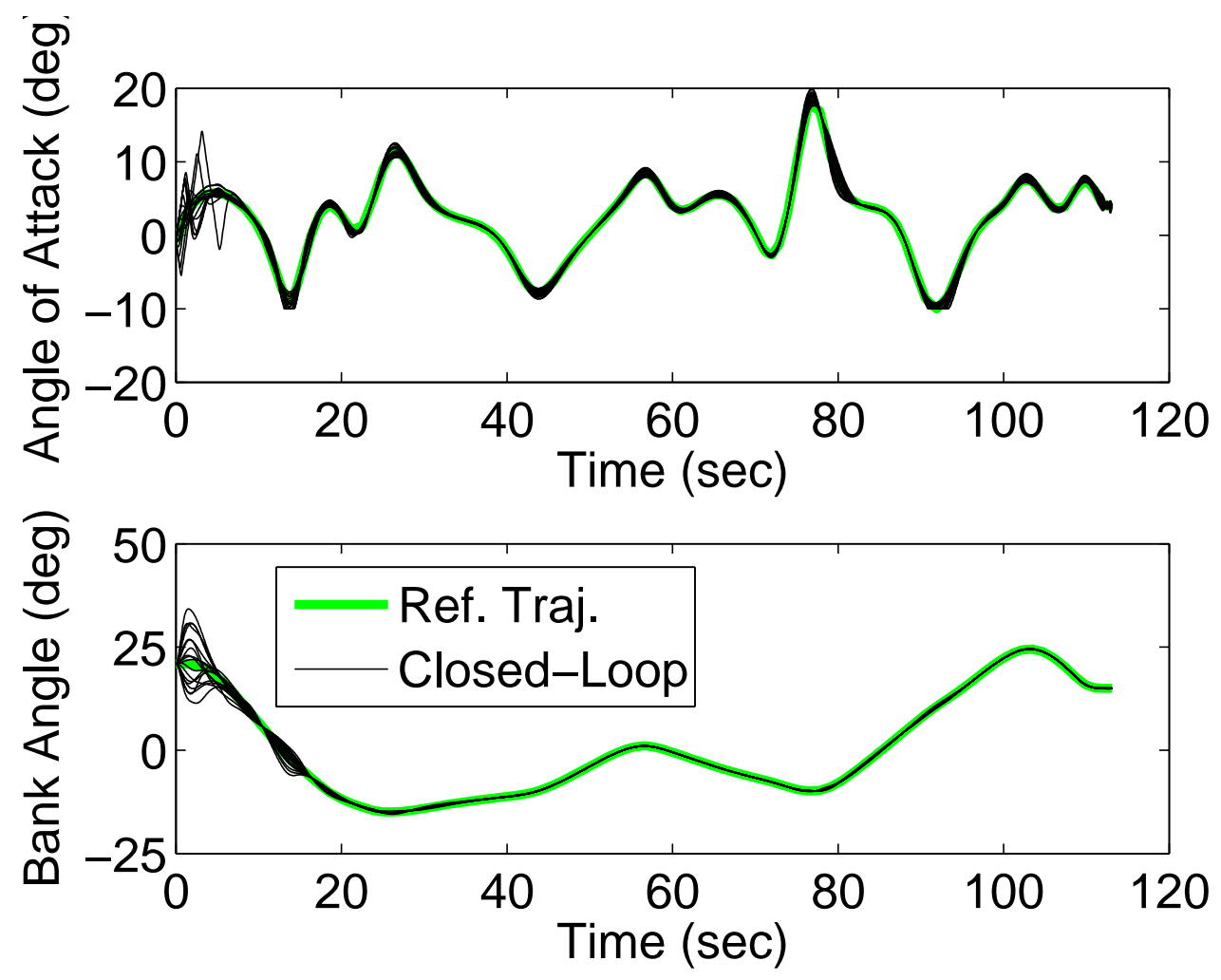

Figure 4: Closed loop angle of attack and bank angle. 


\section{Conclusions}

An approach for planning terrain following and collision avoidance trajectories for military aircraft has been developed. By solving a minimax optimal control problem, it is possible for an aircraft to follow complex three dimensional terrain within a $2.28 \mathrm{~m}$ envelope above the set clearance height. By limiting the angle of attack and bank angle rates in the optimal trajectories, it is possible to obtain more realistic trajectories in the sense that an aircraft has a finite time, rigid body response. Closed loop trajectories were determined using a receding horizon control strategy and demonstrate excellent performance despite initial errors and parameter uncertainty in the aerodynamic models.

\section{References}

[1] Barfield, F., Probert, J., and Browning, D., All terrain ground collision avoidance and maneuvering terring following for automated low level night attack, IEEE Aerospace \& Electronic Systems Magazine, 8, 1993, 40-47. C696

[2] Menon, P. K. A., Kim, E., and Cheng, V. H. L., Optimal trajectory synthesis for terrain-following flight, Journal of Guidance, Control, and Dynamics, 14, 1991, 807-813. C696

[3] Lu, P., Optimal aircraft terrain-following analysis and trajectory generation, Journal of Guidance, Control, and Dynamics, 18, 1995, 555-560. C696

[4] Bryson, A. E., Desai, M. N., and Hoffman, W. C., The energy-state approximation in performance estimation of supersonic aircraft, Journal of Aircraft, 6, 1969, 481-487. C698 
[5] Bryson, A. E. Dynamic Optimization. Addison-Wesley. Menlow Park, 1999. C698

[6] Williams, P., User's guide to DIRECT Version 1.17, Technical Report, March, 2005. C701

[7] Williams, P., A Gauss-Lobatto quadrature approach for solving optimal control problems, 7th Biennial Engineering Mathematics and Applications Conference, Melbourne, Australia, Sept. 25-28, 2005. C701

[8] De Boor, C., A Practical Guide to Splines. Springer-Verlag, New York, 1978. C702 